\title{
BJR \\ Ultrasound-targeted simvastatin-loaded microbubble destruction promotes OA cartilage repair by modulating the cholesterol efflux pathway mediated by PPARy in rabbits
}

\section{X.Wang, \\ D. Wang, \\ P. Xia, \\ K. Cheng, \\ Q.Wang, \\ X.Wang, \\ Q. Lin, \\ J.Song, \\ A. Chen, \\ X. Li}

From Nanjing First Hospital, Nanjing

Medical University, Nanjing, China
Correspondence should be sent to Xueping Li; email: lixueping6504@163.com

doi: $10.1302 / 2046-3758.1010$ BJR-2021-0162.R3

Bone Joint Res 2021;10(10):693703.

Aims

To evaluate the effect of ultrasound-targeted simvastatin-loaded microbubble destruction $\left(U T M D_{s v}\right)$ for alleviation of the progression of osteoarthritis $(O A)$ in rabbits through modulation of the peroxisome proliferator-activated receptor (PPARY).

\section{Methods}

In vitro, $\mathrm{OA}$ chondrocytes were treated with ultrasound (US), US-targeted microbubble destruction (UTMD), simvastatin (SV), and UTMD ${ }_{s V}$ on alternate days for four weeks. Chondrocytes were also treated with PPARy inhibitor, PPARy inhibitor+ UTMD ${ }_{s v^{\prime}}$ and UTMD ${ }_{s v^{\circ}}$. The cholesterol efflux rate and triglyceride levels were measured using an assay kit and oil red $O$ staining, respectively. In vivo, the $O A$ rabbits were treated with a single intra-articular injection of UTMD, SV, and UTMD ${ }_{S V}$ every seven days for four weeks. Cartilage histopathology was assessed by safranin-O staining and the Mankin score. Total cholesterol (TC) and high-density lipoprotein-cholesterol (HDL-C) in rabbit knee synovial fluid were detected by enzyme-marker assay. Aggrecan, collagen II, and PPARy expression levels were analyzed by Western blotting (WB).

\section{Results}

In vitro, UTMD $\mathrm{D}_{s v}$ significantly increased the cholesterol efflux rate and aggrecan, collagen II, and PPARy levels in OA chondrocytes; these effects were blocked by the PPARy inhibitor. In vivo, UTMD $s v$ significantly increased aggrecan, collagen II, PPARY, and HDL-C levels, while TC levels and Mankin scores were decreased compared with the UTMD, SV, OA, and control groups.

\section{Conclusion}

UTMD $_{s v}$ promotes cartilage extracellular matrix synthesis by modulating the PPARY-mediated cholesterol efflux pathway in OA rabbits.

Cite this article: Bone Joint Res 2021;10(10):693-703.

Keywords: Osteoarthritis, Simvastatin, Microbubble, Cholesterol efflux, Cartilage repair

\section{Article focus}

- This study investigated the role of ultrasound-targeted simvastatin-loaded microbubble destruction (UTMDSV) in the treatment for osteoarthritis (OA) and explored the underlying mechanisms.

\section{Key messages}

- $\mathrm{UTMD}_{\mathrm{sV}}$ promotes the expression of aggrecan and collagen II, and prevents the degradation of articular cartilage in a rabbit knee OA model.

- UTMD $_{\text {SV }}$ increases the cholesterol efflux rate and attenuates lipid deposition in OA chondrocytes. 


\section{Strengths and limitations}

- Our study shows that UTMD $_{\text {sv }}$ reduces drug sideeffects and improves the efficacy of ultrasound therapy, which highlights a promising therapeutic strategy for OA.

- The exact molecular mechanisms by which UTMD ${ }_{\text {sV }}$ alleviates $O A$ requires further investigation.

\section{Introduction}

Osteoarthritis $(\mathrm{OA})$ is a clinically chronic and degenerative disease of articular cartilage. The main symptoms of $\mathrm{OA}$ are joint pain, stiffness, and dysfunction, which are the main causes of physical disability in the elderly. ${ }^{1}$ It is generally believed that the pathogenesis of $\mathrm{OA}$ is mainly due to the degenerative changes in cartilage caused by chronic joint wear. ${ }^{2}$ As the epidemiological and molecular biological research related to OA has advanced, it has been found that lipid metabolism disorders play a key role in the process of OA cartilage degeneration. ${ }^{3,4}$ Therefore, OA has been considered a metabolic disease.

Cholesterol reverse transport (CRT) represents the process by which excessive cholesterol flows from peripheral cells and combines with high-density lipoprotein (HDL) to form high-density lipoprotein-cholesterol (HDLC), which is then transported to the liver for metabolic excretion. ${ }^{5}$ Several studies have demonstrated cholesterol efflux disorders in OA chondrocytes. This decrease in CRT function leads to excessive accumulation of lipids in chondrocytes, leading to hypertrophy and ossification, which exacerbate the degree of cartilage degeneration in OA. ${ }^{6,7}$ Peroxisome proliferator-activated receptor $Y$ (PPARY) performs an important role in the mechanism by which CRT is regulated. ${ }^{8}$ PPARy knockout rats have severe growth and development defects, and are more likely to suffer from cardiovascular and joint diseases.9-11 These studies suggested that PPARY protects against the development of OA in articular cartilage by affecting cholesterol efflux.

Several studies have shown that statins can delay the degeneration of OA chondrocytes by activating PPARY and promoting cholesterol efflux. ${ }^{12-14}$ Therefore, statins are expected to change the course of $\mathrm{OA}$, although longterm oral administration can lead to severe side effects such as abnormal blood sugar levels, liver enzyme abnormalities, myopathy, and memory impairment. Choosing an appropriate route of administration, and shortening the course of treatment to reduce side effects and enhance the efficacy of statins, is a focus of future research.

Ultrasound-targeted microbubble destruction (UTMD) has become a promising clinical technology. It involves the delivery of microbubble contrast agents carrying drugs via peripheral veins or local injection followed by the application of ultrasound (US) when flowing through the target site. US produces cavitation that disrupts the microbubbles, which increases the cell permeability at the target site and promotes the rapid release and delivery of drugs, thereby enhancing the therapeutic effect. ${ }^{15}$
Currently, research into the application of this technology is focused mainly on the fields of cardiovascular disease, and tumour and stem cell therapy. ${ }^{16}$ Although a few studies have confirmed that UTMD can be used for the treatment of rheumatoid arthritis, ${ }^{17,18}$ there are no relevant studies investigating the application of US-targeted simvastatin-loaded microbubble destruction $\left(U T M_{\text {SV }}\right.$ ) for the treatment of OA. In this study, we explored the effect and mechanism of UTMD ${ }_{S V}$ on OA cartilage degeneration by modulating the PPARY-mediated cholesterol efflux pathway.

\section{Methods}

Ethical statement. The experimental protocol relating to rabbits was in accordance with the USA National Institutes of Health's Guidelines of Laboratory Animal Use and approved by the hospital. We adhered to the ARRIVE Guidelines and have included the appropriate ARRIVE checklist.

OA model establishment in rabbits. A total of 41 male New Zealand white rabbits ( six weeks, $2.5 \mathrm{~kg}$ to $3.0 \mathrm{~kg}$ ) were raised in separate cages, with ad libitum access to food and water. Overall, 34 rabbits underwent anterior cruciate ligament transection (ACLT) surgery of the right knee to establish the OA model. ${ }^{19}$ The remaining seven rabbits were treated as control groups by only cutting the knee joint capsule. After surgery, penicillin $(200,000$ IU) was delivered via intramuscular injection twice a day for three consecutive days.

The isolation and culture of chondrocytes. Rabbit chondrocytes from the OA model group $(n=10)$ and control group $(n=1)$ were isolated from the surface of the femoral condyle joint and tibial plateau, and cells from each rabbit were divided into three groups. The cartilage tissue was minced aseptically and then digested with trypsin and type II collagenase (Gibco, USA). After digestion, chondrocytes were seeded in T25 flasks $\left(5 \times 10^{6}\right)$ in Dulbecco's modified Eagle's medium (DMEM) supplemented with penicillin $(50 \mathrm{U} / \mathrm{ml}), 10 \%$ fetal bovine serum (FBS), and streptomycin and cultured at $37^{\circ} \mathrm{C}$ under $5 \%$ $\mathrm{CO}_{2}$. At $80 \%$ to $90 \%$ confluence, the chondrocytes were subcultured in a six-well plate $\left(2 \times 10^{6}\right.$ cells/well) for use in experiments.

Preparation of simvastatin-loaded microbubbles. Simvastatin-loaded microbubbles were customized in Nanjing Tranmedical Biosciences (China). A mixture of dipalmitoylphosphatidylcholine, cholesterol, distearoylp hosphatidylethanolamine-polyethylene glycol 2000, and a-tocopherol lipids was prepared at a ratio of 3:1:1:0.004 in aseptic conditions. Simvastatin was added to the lipid mixture $(0.1 \mathrm{mg} / \mathrm{ml})$. The mixture was then dried in a rotary evaporator flask and a uniform lipid film produced by steam equipment (RE-3002, RuiDe, China). The suspension was transferred to a fresh vial filled with C3F8 gas, which was vibrated using a mechanical oscillator (LY-YKZ-04, JTLIANGYOU, China) to form $0.1 \mathrm{mg} / \mathrm{ml}$ simvastatin microbubbles. The remaining space in the vial 
was filled with high-pressure C3F8 gas and sealed, then stored at $4{ }^{\circ} \mathrm{C}$ for later use. The microbubbles were spherical with a mean diameter of $1,221.9 \mathrm{~nm}$.

In vitro UTMD $_{\text {sv }}$ intervention experiment. For optimization, the chondrocytes $\left(2 \times 10^{6}\right)$ were treated with US (Artison Corporation 74036, USA) at different intensities $\left(0 \mathrm{~W} / \mathrm{cm}^{2}, 0.3 \mathrm{~W} / \mathrm{cm}^{2}, 0.6 \mathrm{~W} / \mathrm{cm}^{2}\right.$, and $\left.1.0 \mathrm{~W} / \mathrm{cm}^{2}\right)$ and different concentrations of simvastatin-loaded microbubbles $(1 \mu \mathrm{M}, 10 \mu \mathrm{M}$, and $100 \mu \mathrm{M})$. This preliminary experiment showed that $1 \mu \mathrm{M}$ simvastatin-loaded microbubbles produced the best effect under the parameters of $1 \mathrm{MHz}$ and $0.3 \mathrm{~W} / \mathrm{cm}^{2}$ US irradiation for 30 seconds. Subsequently, the chondrocytes were treated as follows: 1) Control group-unloaded microbubbles without US; 2 ) US group - US irradiation ( $\left.1 \mathrm{MHz}, 0.3 \mathrm{~W} / \mathrm{cm}^{2}\right)$ for $30 \mathrm{sec}$ onds; 3) UTMD group-unloaded microbubbles under 1 $\mathrm{MHz}, 0.3 \mathrm{~W} / \mathrm{cm}^{2}$ US for 30 seconds; 4) SV group-1 $\mu \mathrm{M}$ simvastatin; and 5) UTMD ${ }_{s V}$ group-1 $\mu \mathrm{M}$ simvastatinloaded microbubbles under $1 \mathrm{MHz}, 0.3 \mathrm{~W} / \mathrm{cm}^{2}$ US for 30 seconds. All groups were treated once every two days for four weeks.

In vitro PPARy inhibitor intervention. In the preliminary experiments, we explored the effects of different concentrations of PPARy inhibitor (T0070907, Selleckchem, USA) on extracellular matrix proteins in chondrocytes. Finally, $12.5 \mu \mathrm{M}$ T0070907 was selected for use in subsequent experiments. The chondrocytes were seeded in six-well plates $\left(2 \times 10^{6}\right.$ cells/well $)$ and treated as follows: 1) Control group - $0.1 \%$ DMSO; 2) PPARy inhibitor group - treated with $12.5 \mu \mathrm{M}$ T0070907 for 48 hours at $37^{\circ} \mathrm{C}$; 3) PPARy inhibitor+ UTMD ${ }_{s V}$ group - treated with $12.5 \mu \mathrm{M}$ T0070907 and $1 \mu \mathrm{M}$ simvastatin-loaded microbubbles under US ( $\left.1 \mathrm{MHz}, 0.3 \mathrm{~W} / \mathrm{cm}^{2}\right)$ for 30 seconds, and continued cultivation for 48 hours at $\left.37^{\circ} \mathrm{C} ; 4\right) \mathrm{UTMD}_{s V}$ grouptreated with $1 \mu \mathrm{M}$ simvastatin-loaded microbubbles under US ( $\left.1 \mathrm{MHz}, 0.3 \mathrm{~W} / \mathrm{cm}^{2}\right)$ for 30 seconds and continued cultivation for 48 hours at $37^{\circ} \mathrm{C}$.

In vivo intra-articular injection experiment. The $\mathrm{OA}$ model rabbits were randomly divided into four groups using a random number table method ( $n=6 /$ group) based on the following treatments: OA group, UTMD group, SV group, and UTMD $S V$ group. The sham operation rabbits were used as a control group $(n=6)$. The treatments were delivered by injection into the right knee joint cavity as follows: UTMD group - unloaded microbubbles under US irradiation ( $\left.1 \mathrm{MHz}, 2 \mathrm{~W} / \mathrm{cm}^{2}\right)$ for five minutes; SV group - $0.1 \mathrm{mg} / \mathrm{ml}$ simvastatin; UTMD $D_{S V}$ group - $0.1 \mathrm{mg} /$ $\mathrm{ml}$ simvastatin-loaded microbubbles under US irradiation $\left(1 \mathrm{MHz}, 2 \mathrm{~W} / \mathrm{cm}^{2}\right)$ for five minutes; control and OA model groups - equivalent volume of saline. Each group received $0.2 \mathrm{ml} / \mathrm{kg}$. All groups were treated once every seven days for four weeks, and the rabbits were killed by air embolism for histological and WB analyses after treatment.

Immunofluorescence. OA chondrocytes were identified by immunofluorescence staining of aggrecan (MA3-16888, Thermo Fisher Scientific, USA), collagen II (Origene, USA), and PPARy (LifeSpan, USA). ${ }^{20}$ Chondrocytes were fixed with $4 \%$ paraformaldehyde for ten minutes, and then blocked with $1 \%$ bovine serum (BSA) for one hour at room temperature. The chondrocytes were labelled for two hours at $37^{\circ} \mathrm{C}$ with antibodies specific for aggrecan, collagen II, and PPARy at $2 \mu \mathrm{g} / \mathrm{ml}$ in $0.1 \%$ BSA. Chondrocytes were then incubated with FITC-conjugated secondary antibodies (Agrisera, Sweden; 1:200) for one hour at $37^{\circ} \mathrm{C}$. Nuclei were stained with 4'6-diamidino-2-phenylindole (DAPI) (Thermo Fisher Scientific) for five minutes in the absence of light. Following nuclear DAPI counterstaining, the number of positive cells among the total were counted in ten randomly selected fields per culture dish.

Western blot analysis. Total protein was extracted using lysis buffer (Keygenbio, China). ${ }^{4}$ After being lysed on ice for 20 minutes, the lysate was centrifuged $(12,000 \mathrm{rpm}$ for 15 minutes, $4^{\circ} \mathrm{C}$ ) and the supernatant was collected. The protein concentration was analyzed using a bicinchoninic acid protein assay kit (Keygenbio), and the remaining protein was mixed with $5 \times$ sodium-dodecyl sulfate-polyacrylamide gel electrophoresis (SDS-PAGE) buffer and heated at $100^{\circ} \mathrm{C}$ for 15 minutes. Proteins were then separated by SDS-PAGE and electroblotted onto polyvinylidene fluoride (PVDF) membranes. All membranes were blocked with $5 \%$ skimmed milk at room temperature for one hour. Membranes were incubated overnight at $4^{\circ} \mathrm{C}$ with the following primary detection antibodies: anti-aggrecan (Thermo Fisher Scientific, 1:1,000), anticollagen II (Origene, 1:1,000), and anti-PPARy (LifeSpan, $1: 1,000)$. Membranes were subsequently washed three times with Tween-Tris buffered saline (TBST) and incubated with secondary detection antibodies (Keygenbio) at $37^{\circ} \mathrm{C}$ for two hours. After washing as described previously, the proteins were visualized using an enhanced chemiluminescence reagent and quantified using the Image 4000 system (Tanon 6600, China). Glyceraldehyde-3phosphate dehydrogenase (GAPDH) (Abcam, USA) was quantified as an endogenous control protein. Relative target protein expression was calculated as a ratio of the expression of GAPDH.

Cholesterol efflux analysis. The cholesterol efflux from chondrocytes was measured using a fluorometric cholesterol efflux assay kit (Sigma-Aldrich, USA). ${ }^{21}$ Briefly, the chondrocytes were seeded in 96-well plates at a density of $2 \times 10^{4}$ cells/well and allowed to adhere overnight at $37^{\circ} \mathrm{C}$ under $5 \% \mathrm{CO}_{2}$, after which each group of chondrocytes was washed with FBS-free Roswell Park Memorial Institute (RPMI, Sigma-Aldrich) medium without FBS. The cells were then labelled with cholesterol and incubated for 16 hours at $37^{\circ} \mathrm{C}$ under $5 \% \mathrm{CO}_{2}$. Subsequently, chondrocytes were incubated for five hours $\left(37^{\circ} \mathrm{C}, 5 \% \mathrm{CO}_{2}\right)$ with $100 \mu \mathrm{l}$ of samples acting as cholesterol acceptors; only FBS-free RPMI was added to the blank control wells. The supernatants (medium) were then transferred to a fresh 96-well plate and the fluorescence was measured $(\mathrm{Fm}, \mathrm{Ex} / \mathrm{Em}=482 / 515 \mathrm{~nm})$ using a SpectraMax M3 microplate reader (Molecular Devices, USA). The adherent cells were solubilized by cell lysis buffer to measure the 
fluorescence $(F c, E x / E m=482 / 515 \mathrm{~nm})$. The cholesterol efflux (\%) was calculated according to the following formula:

Cholesterol efflux $(\%)=\mathrm{Fm} /(\mathrm{Fc}+\mathrm{Fm}) \times 100 \%(\mathrm{Fm}=$ fluorescence intensity of the medium; Fc = fluorescence intensity of the cell lysate).

Oil red 0 staining. The triglyceride level in chondrocytes was detected using an oil red O staining kit (Keygenbio). The chondrocytes in each group were seeded in a six-well plate $\left(2 \times 10^{6}\right.$ cells/well) and incubated for 24 hours $\left(37^{\circ} \mathrm{C}\right.$ under $5 \% \mathrm{CO}_{2}$ ). After washing with PBS, the chondrocytes were fixed in 10\% neutral formaldehyde for 30 minutes, and then stained with oil red $\mathrm{O}$ staining solution at $60^{\circ} \mathrm{C}$ for ten minutes. Cells were exposed to decolorizing solutions $A$ and $B$ twice at room temperature. After re-staining with haematoxylin for 20 to 60 seconds, the cells were rinsed with decolorizing solution $B$ and observed under a microscope (Olympus BX43, Japan). The cells in each group were dissolved in isopropanol solution $(500 \mu \mathrm{l} /$ well), and then transferred to a 96-well plate to detect absorbance at $490 \mathrm{~nm}$ using a TECAN Infinite M200PRO microplate reader (Thermo Fisher Scientific).

T-CHO and HDL-C analysis. Synovial fluid was collected from the knee joint of rabbits and placed at room temperature for 30 minutes before centrifugation at 4,000 rpm for five minutes. The total cholesterol (TC) and HDL-C levels were determined using an oxidase-peroxidase coupling (COD-PAP) test kit (JianCheng Bioengineering, China). The absorbance was measured at $510 \mathrm{~nm}$ and $546 \mathrm{~nm}$ using a TECAN Infinite M200PRO microplate reader (Thermo Fisher Scientific).

Histological analysis. Histological analysis of articular cartilage was performed for all groups. ${ }^{22}$ The femoral condyles and tibial plateau of the right knees were fixed in $10 \%$ formaldehyde for three days. The tissues were then sectioned along the sagittal plane, embedded in paraffin, and sectioned after decalcification. Sections were stained with fresh Weigert iron haematoxylin for five minutes, and then washed in running tap water. After soaking for 15 seconds in acid alcohol differentiation solution ( $1 \%$ hydrochloric acid, 70\% alcohol), slides were washed with distilled water for ten minutes before immersion in fast green stain for five minutes. Next, the sections were washed briefly with $1 \%$ acetic acid for 15 seconds to remove residual fast green stain. Subsequently, sections were stained with safranin-O for five minutes, dehydrated with 95\% alcohol, cleared with xylene, and finally sealed in optical resin. The following characteristics of the sections prepared from each group were graded according to Mankin's scoring principles: the tide-line integrity, the number and structure of chondrocytes, and dye intensity. The severity of tissue lesions was graded and standardized as a scoring criterion, with higher scores reflecting greater severity of the lesion. The sections were evaluated independently by two observers (XW, DW) using a blinded method, and average scores were calculated as the result.
Statistical analysis. For the in vitro study, three samples of chondrocytes were extracted from one rabbit (total 11 rabbits), and for the in vivo study, the cartilage samples were extracted from six rabbits of each group (total 30 rabbits). Each chondrocyte and cartilage sample was repeatedly tested three times. All normally distributed data were expressed as means and standard deviations (SDs). Independent-samples t-tests and one-way analysis of variance (ANOVA) followed by least significant difference (LSD) tests were used to evaluate the significant differences between the groups. All statistical analysis was performed using SPSS software (version 21.0, IBM, USA). Statistical significance was set at $p<0.05$.

\section{Results}

Cholesterol efflux and extracellular matrix proteins decrease in OA chondrocytes. To test the hypothesis that extracellular matrix (ECM) protein is lost and disruption of cholesterol metabolism occurs in OA chondrocytes, we established the rabbit OA model by ACLT surgery. Western blot analysis confirmed that the expression levels of aggrecan ( $p=0.003$, independent-samples t-test), collagen II ( $p=0.003$, independent-samples t-test), and PPARy ( $p=0.004$, independent-samples $t$-test) were significantly reduced in the OA group compared with those in the control group (Figures $1 \mathrm{a}$ and $1 b)$. The cholesterol efflux rate was also decreased ( $p=$ 0.002 , independent-samples $t$-test) in OA chondrocytes (Figure 1c). Immunofluorescence analysis of the expression of aggrecan ( $p=0.002$, independent-samples $t$-test), collagen II ( $p<0.001$, independent-samples $t$-test), and PPARY ( $p<0.001$, independent-samples $t$-test) showed that the expression of these proteins was significantly reduced in the OA group compared with the control group (Figures $1 \mathrm{~d}$ and 1e).

UTMD $_{s v}$ increases cholesterol efflux and extracellular matrix proteins levels in OA chondrocytes. To investigate the effect of UTMD ${ }_{S V}$ on ECM expression and cholesterol metabolism in OA chondrocytes, we treated OA chondrocytes with US, UTMD, SV, and UTMD ${ }_{S V}$. Western blot analysis showed that the expression levels of aggrecan ( $p$ $<0.001$, one-way ANOVA), collagen II ( $p<0.001$, oneway ANOVA), and PPARy ( $p<0.001$, one-way ANOVA) were significantly upregulated in the UTMD ${ }_{S V}$ group compared with those in the OA group. Compared with the US or SV groups, aggrecan ( $p<0.001$; $p<0.001$, one-way ANOVA), collagen II $(p<0.001$; $p<0.001$, oneway ANOVA) and PPARY ( $<<0.001 ; p<0.001$, one-way ANOVA) expression levels were significantly increased in the UTMD $_{S V}$ group (Figures $2 \mathrm{a}$ to $2 \mathrm{c}$ ). Triglyceride levels in chondrocytes were analyzed by spectrophotometric analysis of oil red $\mathrm{O}$ staining (Figures $2 \mathrm{e}$ and $2 \mathrm{f}$ ). Compared with the OA group, the absorbance was significantly decreased $(p=0.027 ; p=0.005 ; p<0.001$, one-way ANOVA)

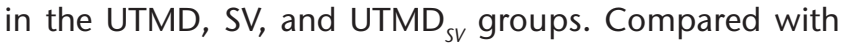
the UTMD or SV groups, the absorbance was significantly reduced $(p=0.004 ; p=0.023$, one-way ANOVA) in the 


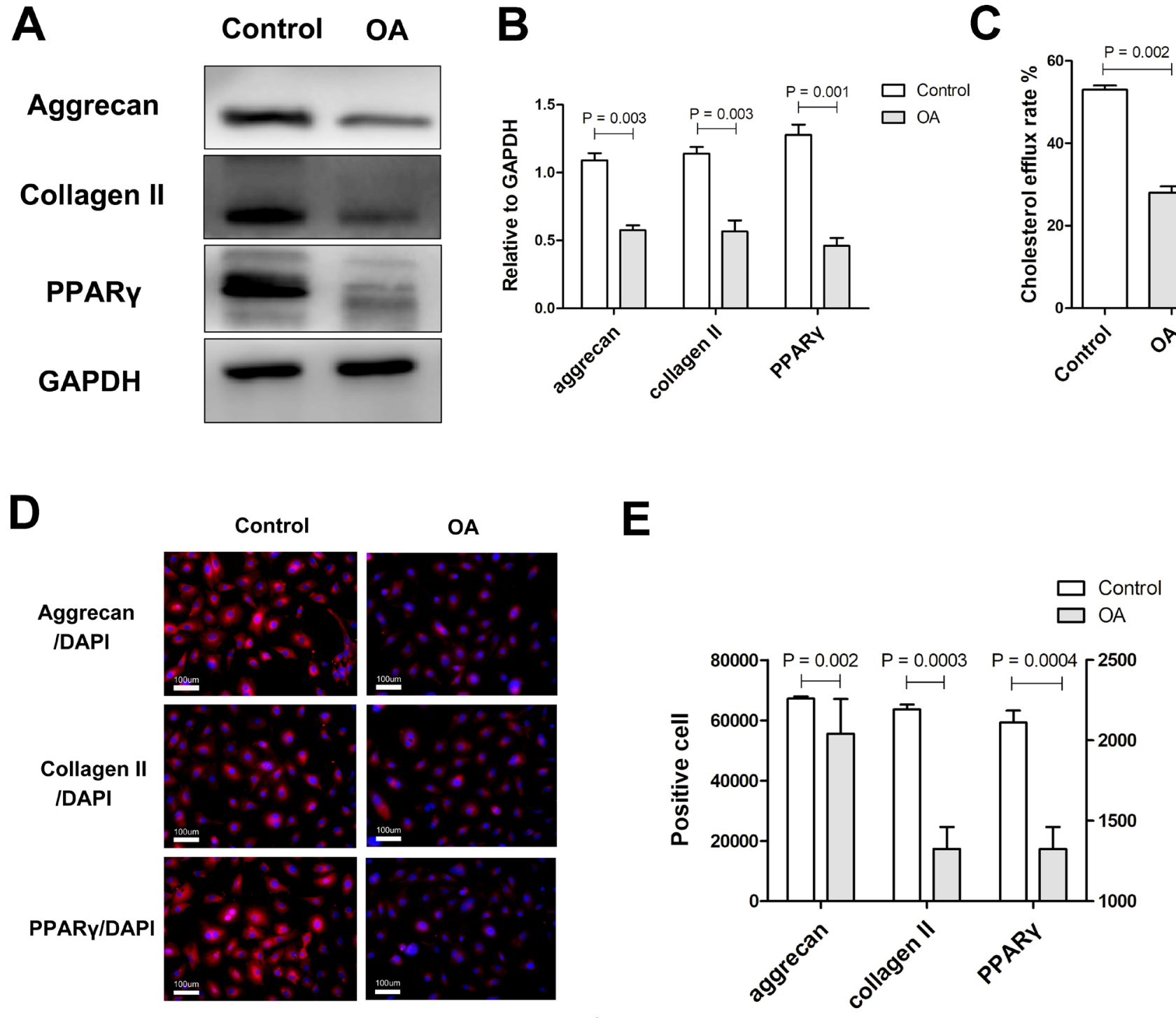

Fig. 1

The extracellular matrix expression and cholesterol efflux rate decreases in osteoarthritis (OA) chondrocytes. a) and b) Western blot analysis of aggrecan, collagen II, and peroxisome proliferator-activated receptor (PPARy) expression in chondrocytes. Glyceraldehyde 3-phosphate dehydrogenase (GAPDH) was used as the endogenous control. c) The difference in cholesterol efflux rate between the control and osteoarthritis (OA) groups. d) and e) Representative immunofluorescence staining and quantification of the positive cell numbers. Chondrocytes are stained blue and the target proteins are stained red (scale bar $=100 \mu \mathrm{m}$ ). DAPI, 4',6-diamidino-2-phenylindole. All p-values calculated using independent-samples t-test.

UTMD $_{s V}$ group. To determine if UTMD ${ }_{s V}$ affects cholesterol metabolism, we further analyzed cholesterol efflux in each group (Figure $2 \mathrm{~g}$ ). Compared with the OA group, cholesterol efflux was significantly increased ( $p=0.001$; $p<0.001 ; p<0.001$, one-way ANOVA) in the UTMD, $\mathrm{SV}$, and $\mathrm{UTMD}_{S V}$ group respectively. Compared with the UTMD group, the cholesterol efflux level was increased ( $p$ $=0.001$, one-way ANOVA) in the UTMD ${ }_{s V}$ group.

The expression level of extracellular matrix proteins after PPARY inhibitor and UTMDsv intervention. To investigate the involvement of PPARy in the process of OA cartilage ECM expression and cholesterol efflux rate, we treated chondrocytes with a PPARy inhibitor (12.5 $\mathrm{MM}$ T0070907). Compared with the control group, the expression levels of aggrecan $(p<0.001)$, collagen II $(p<$
$0.001)$, and PPARy $(p<0.001)$ and the cholesterol efflux rate $(p=0.002)$ were significantly decreased in the PPARy inhibitor group (Figures $3 a$ to $3 e$ ). Further investigation of the effect of UTMD ${ }_{S V}$ on chondrocytes showed that aggrecan $(p<0.001)$, collagen II $(p<0.001)$, and PPARY $(p$ $<0.001)$ expression levels and the cholesterol efflux rate $(p<0.001)$ were significantly increased in the UTMD group compared with those in the PPARy inhibitor groups (Figures $3 a$ to $3 e$ ); all p-values were calculated using oneway ANOVA.

Pathology score and cholesterol level after UTMD ${ }_{s v}$ intervention. We further investigated the activity of aggrecan, collagen II, and PPARY in vivo after intra-articular injection of UTMD, SV, or UTMD ${ }_{S V}$. Safranin-O staining (Figures $4 \mathrm{a}$ and $4 \mathrm{~b}$ ) showed that the Mankin scores were increased 
A

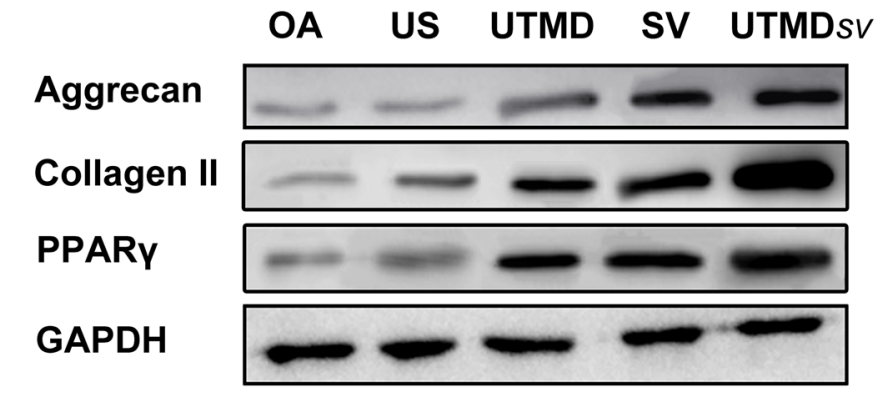

B
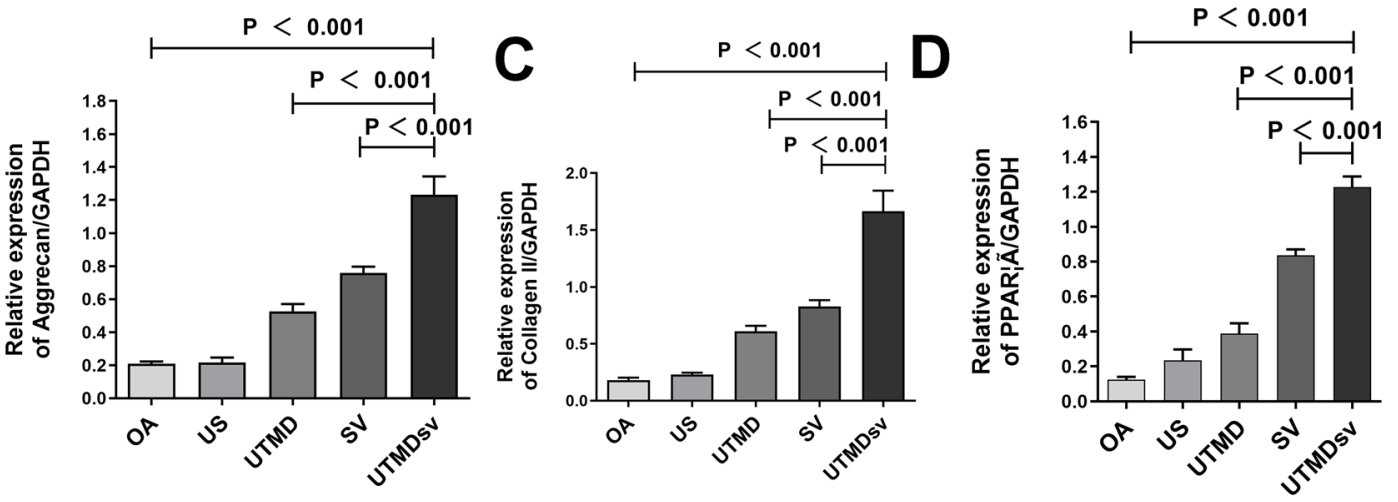

E

OA

US

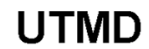

SV

UTMDsv
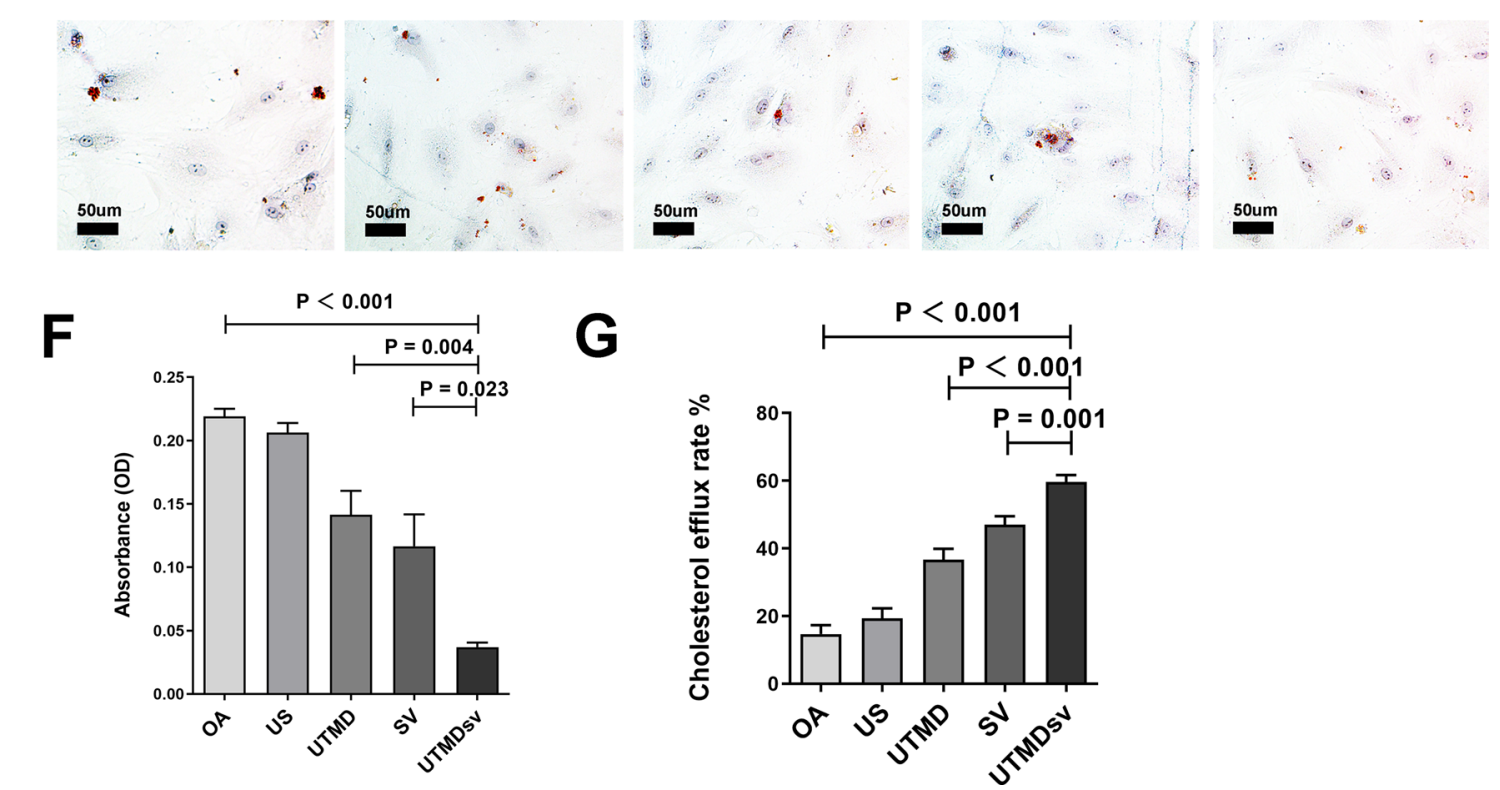

Fig. 2

Effects of UTMD $\mathrm{Sv}_{s v}$ on cartilage metabolism and lipid metabolism. a) to d) Representative Western blot and quantification of aggrecan, collagen II, and PPARY, relative to glyceraldehyde-3-phosphate dehydrogenase (GAPDH) in osteoarthritis (OA) chondrocytes after treatment with ultrasound (US), US-targeted microbubble destruction (UTMD), simvastatin (SV), and US-targeted simvastatin-loaded microbubble destruction (UTMD sv ). e) Representative oil red images; scale $b a r=50 \mu \mathrm{m}$. f) Triglyceride levels were quantified in chondrocytes by measuring absorbance at $490 \mathrm{~nm}$. g) The difference in cholesterol efflux rate between groups.

$(p<0.001)$ in the OA group compared with those in the control group. Compared with the SV group, the Mankin scores were decreased $(p=0.044)$ in the UTMD group. Enzymatic analysis levels of TC and HDL-C levels in synovial fluid showed that the TC level was increased $(p<$ 0.001 ) in the OA group compared with that in the control group. Compared with the OA group, TC levels were reduced $(p<0.001 ; p<0.001)$ in the SV and UTMD ${ }_{s V}$ groups. 

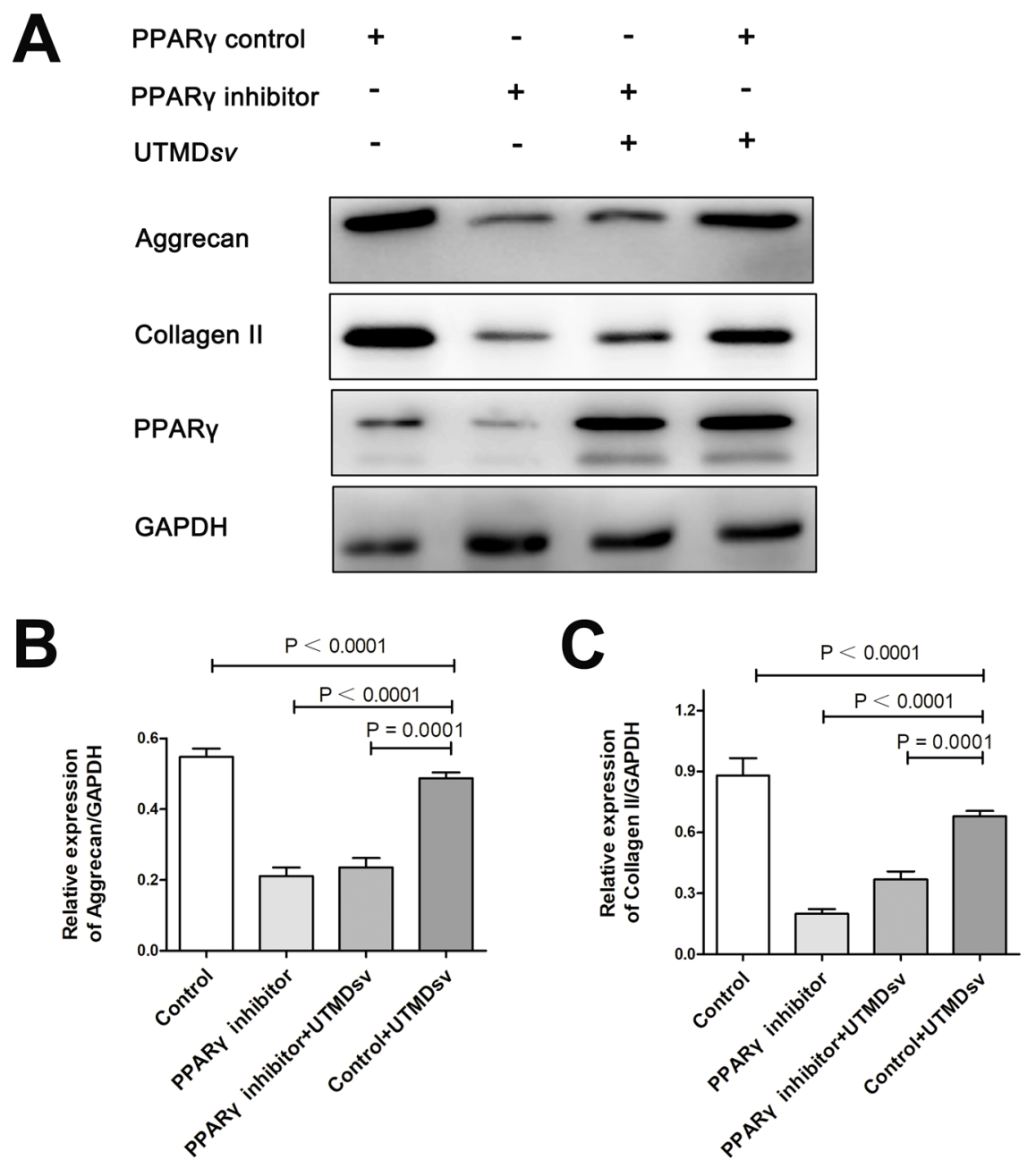

D

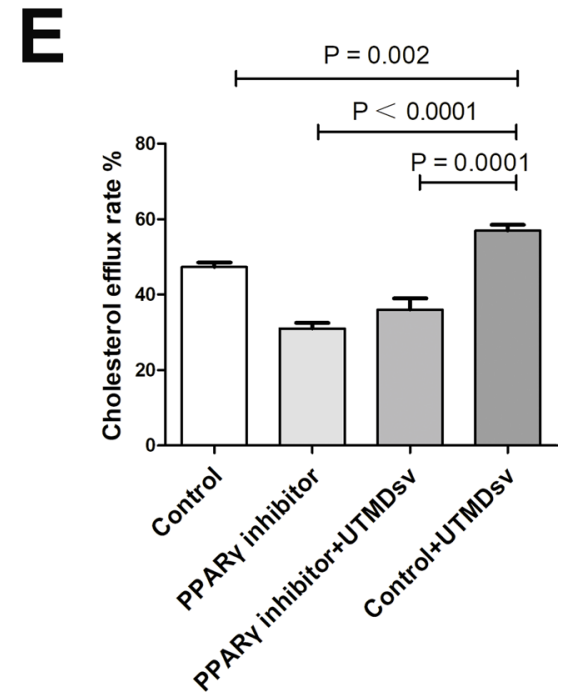

Fig. 3

Peroxisome proliferator-activated receptor (PPARy) inhibitor reduces the extracellular matrix production and the cholesterol efflux rate in chondrocytes. The chondrocytes were treated with PPARY inhibitor. a) to d) Western blot and quantification of the relative expression levels of aggrecan, collagen II, and PPARY after PPARY inhibitor and ultrasound-targeted simvastatin-loaded microbubble destruction $\left(\mathrm{UTMD}_{S \mathrm{~S}}\right)$ intervention in osteoarthritis (OA) chondrocytes; glyceraldehyde 3-phosphate dehydrogenase (GADPH) was used as the endogenous control. e) The effect of the PPARY inhibitor and UTMD ${ }_{S V}$ on the cholesterol efflux rate. All p-values were calculated using one-way analysis of variance. 
A Control

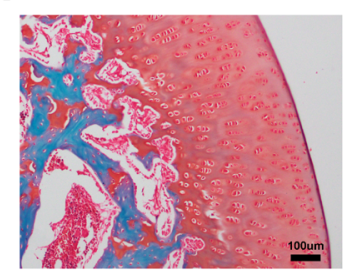

OA

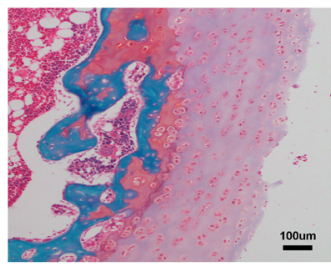

UTMD

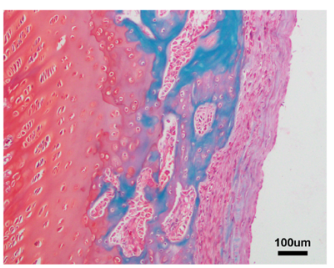

SV

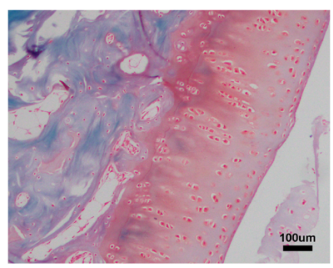

UTMDsv

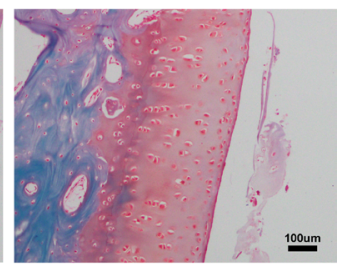

B

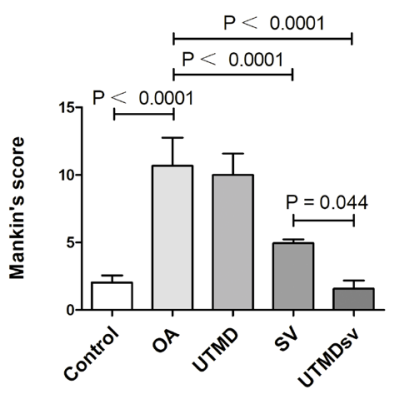

C

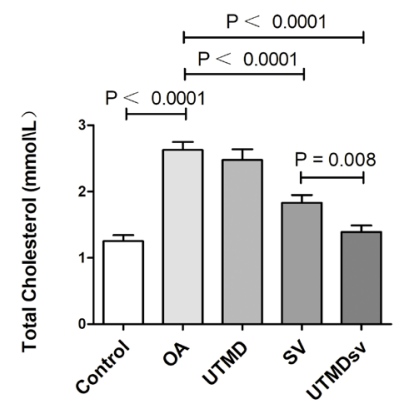

D

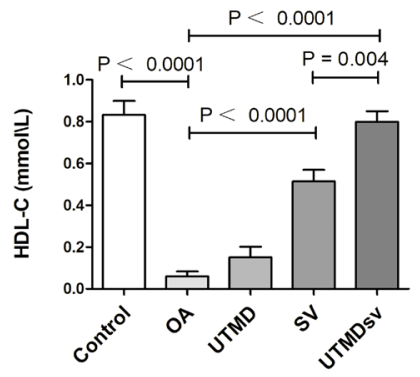

$E$

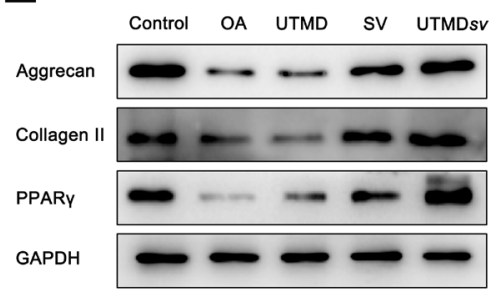

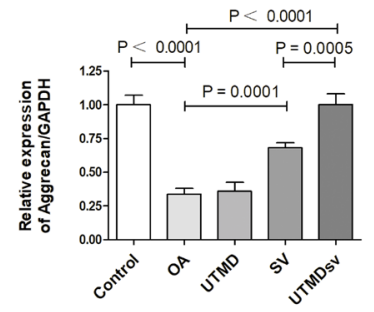

Fig. 4
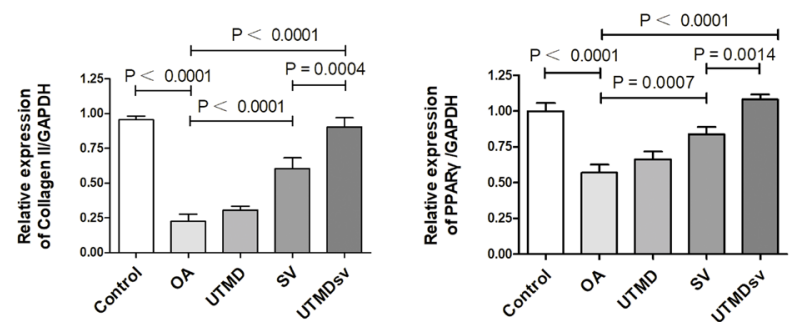

Effects of ultrasound-targeted simvastatin-loaded microbubble destruction (UTMD ${ }_{s v}$ ) on cholesterol regulation and cartilage metabolism in osteoarthritic (OA) rabbit. Animals were respectively treated with UTMD, SV, and UTMD ${ }_{S V}$ every seven days for four weeks. Equivalent saline was administered into the control and OA groups. a) and b) The representative safranin-O images and Mankin scoring of OA parameters $(n=3)$. c) and d) The levels of total cholesterol (TC) and high-density lipoprotein cholesterol (HDL-C) in rabbit knee synovial fluid were detected by an enzyme-marker assay. e) Western blot and quantification of aggrecan, collagen II, and peroxisome proliferator-activated receptor (PPARY) relative to glyceraldehyde 3-phosphate dehydrogenase (GADPH) ( $=3$ ).

Compared with the SV group, TC levels were reduced ( $p$ $=0.008$ ) in the UTMD ${ }_{s V}$ group (Figure $4 \mathrm{c}$ ). Compared with the control group, HDL-C was decreased $(p<0.001)$ in the OA group. Compared with the OA group, HDL-C levels were increased $(p<0.001 ; p<0.001)$ in the SV and UTMD $_{S V}$ groups. Compared with the SV group, HDL-C was increased $(p=0.004)$ in the UTMD ${ }_{s V}$ group (Figure $4 d)$. Aggrecan $(p<0.001)$, collagen II $(p<0.001)$, and PPARy $(p<0.001)$ expression levels were significantly decreased in the OA group compared with those in the control group. Compared with the OA group, aggrecan $(p<0.001 ; p<0.001)$, collagen II $(p<0.001 ; p<0.001)$, and PPARY $(p<0.001 ; p<0.001)$ expression levels were increased in the SV and UTMD ${ }_{s V}$ groups. Compared with the SV group, the expression levels of these proteins were increased $(p<0.001 ; p<0.001 ; p<0.001)$ in the UTMD group (Figure 4e); all $p$-values calculated using one-way ANOVA.

The mechanism of the cholesterol efflux pathway mediated by PPARY in chondrocytes. As shown in Figure 5, UTMD promotes excessive cholesterol deposition in OA chondrocytes, which combines with HDL to form HDL-C and is subsequently transported to the liver for metabolism, a process mediated by PPARy. In addition, PPARy also activates the expression of aggrecan and collagen II in chondrocytes to achieve the purpose of cartilage protection.

\section{Discussion}

In this study, we investigated the ability of $\mathrm{UTMD}_{S V}$ to regulate the cholesterol efflux rate through PPARY and delay the degeneration of rabbit OA cartilage. We found that UTMD ${ }_{S V}$ increased the expression of PPARy, aggrecan, and collagen II in chondrocytes, promoted the cholesterol efflux rate, and regulated cholesterol metabolism in the joint fluid, thereby delaying the process of OA cartilage degeneration.

A previous study has shown that aggrecan and collagen II, major ECM proteins, are degraded in the development of $\mathrm{OA}^{23}$ In the present study, we used immunofluorescence staining to show that the expression of aggrecan and collagen II in OA chondrocytes was decreased, indicating damage to the surface of OA cartilage. We showed that treatment of OA chondrocytes with UTMD ${ }_{s v}$ activated ECM expression and subchondral bone pathology reconstruction to achieve cartilage repair. 


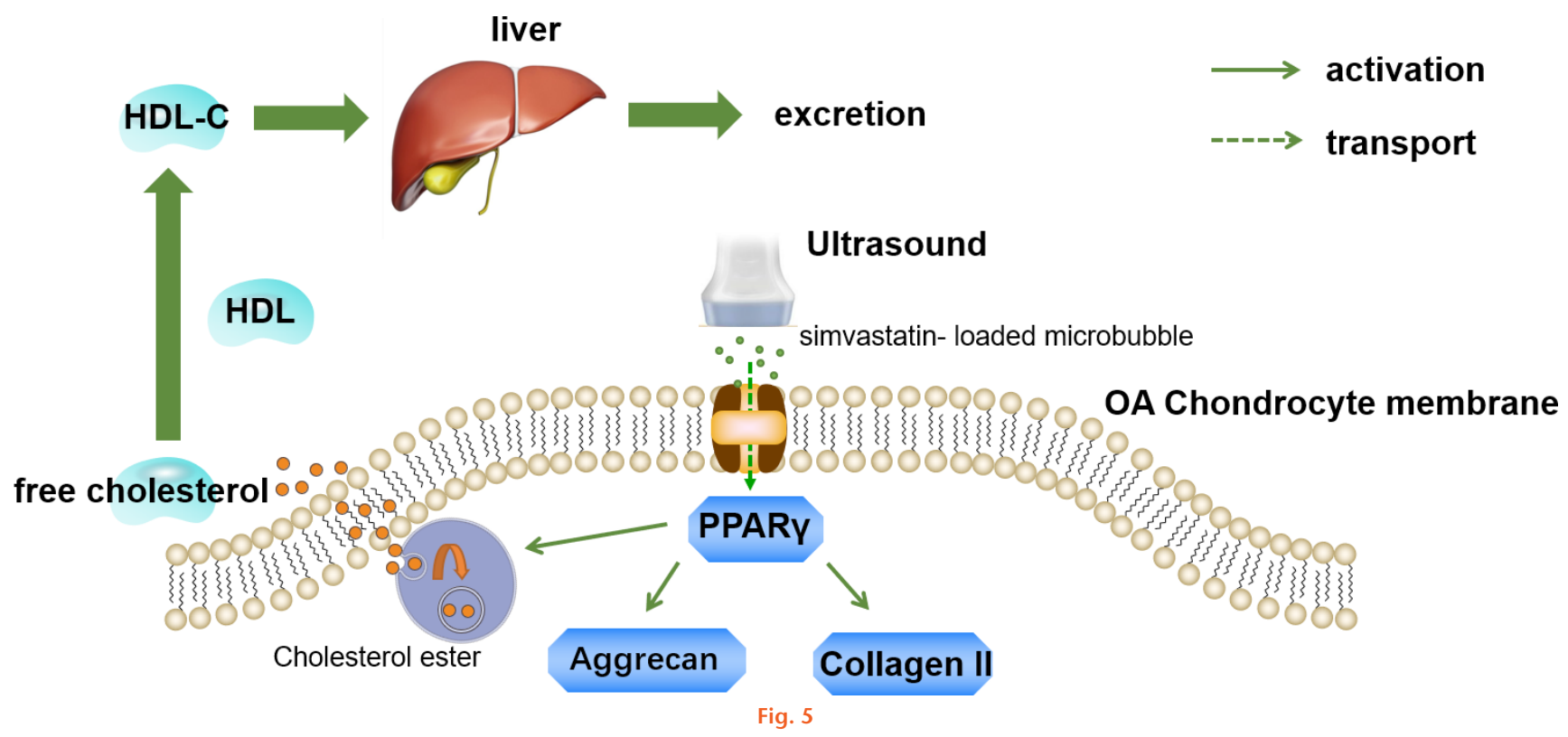

Simplified schematic of the cholesterol efflux pathway mediated by peroxisome proliferator-activated receptor (PPARY) in chondrocytes. HDL, high-density lipoprotein; HDL-C, HDL cholesterol; OA, osteoarthritis.

A superabundance of cholesterol in cells can cause toxicity, leading to cell rupture, apoptosis, or necrosis, and can also degrade aggrecan and collagen II in chondrocytes. ${ }^{724,25}$ High-cholesterol diets cause cartilage degeneration and the occurrence of $\mathrm{OA}$ in a rat experimental model, while reducing cholesterol levels reduces the incidence of cartilage degeneration. ${ }^{26}$ Blood circulation is associated with synovial circulation via the capillaries and lymphatic system, which may account for the high levels of cholesterol in joint fluid. Under such circumstances, chondrocytes are exposed to increased cholesterol levels in the synovial fluid, which causes damage to the cartilage protein in the ECM. Persistently high levels of cholesterol levels in the synovial fluid results in chondrocyte hypertrophy and cartilage ossification, which further exacerbates the severity of OA cartilage degeneration. ${ }^{27,28}$ In our study, we also found that triglycerides were excessively deposited in OA chondrocytes, and the cholesterol efflux rate was decreased. This is consistent with previous research. ${ }^{7}$

PPARy plays a key role in CRT by promoting the cholesterol efflux rate and maintaining the dynamic balance of cholesterol metabolism..$^{29,30}$ One study has shown that PPARY affects chondrocyte metabolism and lipid metabolism, and participates in the occurrence and development of OA; direct activation of PPARY can promote cholesterol efflux and reduce lipid deposition in chondrocytes. $^{29}$ In our study, we found that the cholesterol efflux rate decreased significantly after inhibiting PPARy, and the cholesterol efflux rate increased after the PPARY combined with UTMD ${ }_{S V}$ intervention. These findings indicate that PPARY is a key factor affecting cholesterol efflux, and that UTMD sV not only improves the cholesterol efflux rate effectively but also increases the expression of aggrecan and collagen II in chondrocytes.

Simvastatin promoted cholesterol efflux by activating PPARy, reducing lipid deposition, and upregulating expression of the ECM, thus protecting cartilage. ${ }^{13,14}$ Several studies have shown that statins not only reduce blood lipids effectively, ${ }^{31}$ but also protect against chondrocyte ageing and degeneration and prevent ECM degradation. These functions were shown to protect articular cartilage, reducing the overall rate of knee OA progression by $50 \% .{ }^{32-36}$ However, a clinical study showed that long-term use of statins can cause myopathy, liver dysfunction, diabetes, and cerebral haemorrhage. ${ }^{37,38}$ To avoid the adverse reactions caused by oral statins, some studies explored the effect of intra-articular injection of different simvastatin concentrations $(0.01 \mathrm{mg} / \mathrm{ml}, 0.1 \mathrm{mg} /$ $\mathrm{ml}$, and $0.5 \mathrm{mg} / \mathrm{ml}$ ) on early OA cartilage degeneration in rabbits. The results showed that $0.5 \mathrm{mg} / \mathrm{ml}$ simvastatin has a significantly protective effect on early rabbit OA. ${ }^{39,40}$ Previous literature and our research results have shown that abnormal cholesterol efflux mainly exists in $\mathrm{OA}$ chondrocytes. In this study, we also explored the effect of simvastatin on the lipid metabolism of OA chondrocytes and found that simvastatin can promote cholesterol efflux from OA chondrocytes. In addition, we found that the injection of $0.1 \mathrm{mg} / \mathrm{ml}$ simvastatin microbubbles into the joint cavity of OA rabbits significantly reduced the Mankin score, while the expression levels of aggrecan, collagen II, and PPARy were significantly increased. The effects of simvastatin on normal chondrocytes were not explored in this study, as our research focused mainly on proving the effect of statins on the abnormal cholesterol efflux of OA chondrocytes. 
UTMD can cause the continuous compression and expansion that induces microbubble rupture, which can improve the efficacy of drugs and reduce the dosage required. ${ }^{41-43}$ Studies have shown that microbubbles can improve the delivery of drugs and reduce the adverse effects caused in the gastrointestinal tract by oral administration. ${ }^{44}$ In addition, UTMD can reduce the required intensity and duration of US and avoid the detrimental effects of US cavitation, such as damage to cell membranes, breakage of DNA chains, inhibition of cell proliferation, promotion of apoptosis, and other adverse reactions ${ }^{45}$ In our study, US alone did not significantly increase the cholesterol efflux rate or reduce TC levels, although we found that low-intensity US combined with statin microbubbles affected cholesterol efflux. We also found that UTMD $D_{S V}$ significantly reduced cholesterol efflux and increased the expression of collagen II and aggrecan. $\mathrm{UTMD}_{s v}$ treatment occurred during the phase of surgeryinduced cartilage degradation. It was not tested whether UTMD $_{S V}$ would have a beneficial effect on established OA. Furthermore, the statins could have affected processes other than just PPARy or other cells within the mixed cell population isolated from $\mathrm{OA}$ rabbit knees.

In summary, our results verified that UTMD $D_{S V}$ promotes the cartilage ECM synthesis in a model of rabbit knee OA. The main mechanism may be related to PPARY-mediated regulation of lipid metabolism, promotion of cholesterol efflux, and elimination of chondrocyte lipids.

\section{Supplementary material}

$\because \quad$ An ARRIVE checklist is included to show that the ARRIVE guidelines were adhered to in this study.

\section{References}

1. Katz JN, Arant KR, Loeser RF. Diagnosis and treatment of hip and knee osteoarthritis: A review. JAMA. 2021;325(6):568-578.

2. Conaghan PG, Kloppenburg M, Schett G, Bijlsma JWJ, EULAR osteoarthritis ad hoc committee. Osteoarthritis research priorities: A report from a EULAR ad hoc expert committee. Ann Rheum Dis. 2014;73(8):1442-1445.

3. Wang $\mathbf{X}$, Hunter $\mathbf{D}, \mathbf{X} \mathbf{J}$, Ding C. Metabolic triggered inflammation in osteoarthritis. Osteoarthritis Cartilage. 2015;23(1):22-30.

4. Si H-B, Zeng Y, Liu S-Y, et al. Intra-articular injection of microrna-140 (mirna-140) alleviates osteoarthritis (OA) progression by modulating extracellular matrix (ECM) homeostasis in rats. Osteoarthritis Cartilage. 2017;25(10):1698-1707.

5. Chawla A, Boisvert WA, Lee $\mathbf{C H}$, et al. A PPAR gamma-lxr-abca1 pathway in macrophages is involved in cholesterol efflux and atherogenesis. Mol Cell. 2001;7(1):161-171.

6. Kostopoulou F, Malizos KN, Papathanasiou I, Tsezou A. Microrna-33a regulates cholesterol synthesis and cholesterol efflux-related genes in osteoarthritic chondrocytes. Arthritis Res Ther. 2015;17:42.

7. Masuko K, Murata M, Suematsu N, et al. A metabolic aspect of osteoarthritis: Lipid as a possible contributor to the pathogenesis of cartilage degradation. Clin Exp Rheumatol. 2009;27(2):347-353.

8. Tosheska Trajkovska K, Topuzovska S. High-density lipoprotein metabolism and reverse cholesterol transport: Strategies for raising HDL cholesterol. Anatol J Cardiol. 2017:18(2):149-154

9. Vasheghani F, Zhang Y, Li Y-H, et al. PPARy deficiency results in severe, accelerated osteoarthritis associated with aberrant $\mathrm{MTOR}$ signalling in the articular cartilage. Ann Rheum Dis. 2015;74(3):569-578.

10. Jiang $\mathbf{T}$, Ren $\mathbf{K}$, Chen $\mathbf{Q}$, et al. Leonurine prevents atherosclerosis via promoting the expression of abca1 and abcg1 in a ppar $\gamma / / \mathrm{xr} \alpha$ signaling pathway-dependent manner Cell Physiol Biochem. 2017;43(4):1703-1717.
11. Tsezou A, Iliopoulos D, Malizos KN, Simopoulou T. Impaired expression of genes regulating cholesterol efflux in human osteoarthritic chondrocytes. J Orthop Res. 2010;28(8):1033-1039.

12. Chang C-H, Hsu Y-M, Chen Y-C, et al. Anti-inflammatory effects of hydrophilic and lipophilic statins with hyaluronic acid against Ips-induced inflammation in porcine articular chondrocytes. J Orthop Res. 2014;32(4):557-565.

13. Tenenbaum A, Fisman EZ. Balanced pan-PPAR activator bezafibrate in combination with statin: Comprehensive lipids control and diabetes prevention? Cardiovasc Diabetol. 2012;11:140.

14. Bayyurt S, Küçükalp A, Bilgen MS, Bilgen ÖF, Çavuşoğlu İ, Yalçınkaya U. The chondroprotective effects of intraarticular application of statin in osteoarthritis: An experimental study. Indian J Orthop. 2015;49(6):665-671.

15. Park D, Ryu H, Kim HS, et al. Sonophoresis using ultrasound contrast agents for transdermal drug delivery: an in vivo experimental study. Ultrasound Med Biol. 2012;38(4):642-650.

16. Roovers S, Segers T, Lajoinie G, et al. The role of ultrasound-driven microbubble dynamics in drug delivery: From microbubble fundamentals to clinical translation. Langmuir. 2019;35(31):10173-10191.

17. Liao AH, Chung HY, Chen W. S, Yeh M. K. Efficacy of combined ultrasoundand-microbubbles-mediated diclofenac gel delivery to enhance transdermal permeation in adjuvant-induced rheumatoid arthritis in the rat. Ultrasound Med Biol. 2016;42(8):1976-1985.

18. Liao AH, Chuang HC, Chung HY. Efficacy of ultrasound mediated microbubbles in diclofenac gel to enhance transdermal permeation in rheumatoid arthritis induced rat. Annu Int Conf IEEE Eng Med Biol Soc. 2015;2015:3521-3524.

19. Jean Y-H, Wen Z-H, Chang Y-C, et al. Increase in excitatory amino acid concentration and transporters expression in osteoarthritic knees of anterior cruciate ligament transected rabbits. Osteoarthritis Cartilage. 2008;16(12):1442-1449.

20. Sheng $\mathbf{P}$, Zhu $\mathbf{H}$, Zhang $\mathbf{W}$, et al. The immunoglobulin superfamily member 3 (igsf3) promotes hepatocellular carcinoma progression through activation of the nf- $\kappa \mathrm{b}$ pathway. Ann Trans/ Med. 2020;8(6):378.

21. Singla R, Soni S, Padwad YS, Acharya A, Yadav SK. Sustained delivery of BSA/ HSA from biocompatible plant cellulose nanocrystals for in vitro cholesterol release from endothelial cells. Int J Biol Macromol. 2017;104(Pt A):748-757.

22. Wang XJ, Zhang ZH, Zhang J, et al. Malfunction of autophagy in tibial growth plate chondrocytes causes increased apoptosis rate in chronic renal insufficiency rats. Zhonghua Yi Xue Za Zhi. 2020;100(2):141-145

23. Zhu G, Oian Y, Wu W, Li R. Negative effects of high mechanical tensile strain stimulation on chondrocyte injury in vitro. Biochem Biophys Res Commun. 2019;510(1):48-52.

24. Litvinov DY, Savushkin EV, Garaeva EA, Dergunov AD. Cholesterol efflux and reverse cholesterol transport: Experimental approaches. Curr Med Chem. 2016;23(34):3883-3908.

25. Sansone V, Applefield RC, De Luca $\mathbf{P}$, et al. Does a high-fat diet affect the development and progression of osteoarthritis in mice?: A systematic review. Bone Joint Res. 2019;8(12):582-592.

26. Gierman LM, van der Ham F, Koudijs A, et al. Metabolic stress-induced inflammation plays a major role in the development of osteoarthritis in mice. Arthritis Rheum. 2012;64(4):1172-1181.

27. Zhu $\mathbf{X}$, Chen $\mathbf{F}$, Lu $\mathbf{K}$, Wei A, Jiang $\mathbf{0}$, Cao W. PPARy preservation via promoter demethylation alleviates osteoarthritis in mice. Ann Rheum Dis. 2019;78(10):1420-1429

28. Fahmi H, Martel-Pelletier J, Pelletier J-P, Kapoor M. Peroxisome proliferatoractivated receptor gamma in osteoarthritis. Mod Rheumatol. 2011;21(1):1-9.

29. Vedhachalam C, Liu L, Nickel M, et al. Influence of ApoA-I structure on the ABCA1-mediated efflux of cellular lipids. J Biol Chem. 2004;279(48):49931-49939.

30. Wang M-D, Franklin V, Marcel YL. In vivo reverse cholesterol transport from macrophages lacking ABCA1 expression is impaired. Arterioscler Thromb Vasc Biol. 2007;27(8):1837-1842.

31. Adams SP, Alaeiilkhchi N, Wright JM. Pitavastatin for lowering lipids. Cochrane Database Syst Rev. 2020;6:CD012735.

32. Yudoh $\mathbf{K}$, Karasawa R. Statin prevents chondrocyte aging and degeneration of articular cartilage in osteoarthritis (OA). Aging (Albany NY). 2010;2(12):990-998.

33. Conaghan PG. The effects of statins on osteoarthritis structural progression: another glimpse of the Holy Grail? Ann Rheum Dis. 2012;71(5):633-634.

34. Dinc M, Bilgen MS, Kucukalp A, Bilgen OF. An assessment of the chondroprotective effects of intra-articular application of statin and tetracycline on early-stage experimental osteoarthritis. ISRN Orthop. 2012;2012:182097. 
35. Bayyurt S, Küçükalp A, Bilgen MS, Bilgen ÖF, Cavuşoğlu İ, Yalçınkaya U. The chondroprotective effects of intraarticular application of statin in osteoarthritis: An experimental study. Indian J Orthop. 2015;49(6):665-671.

36. Simopoulou T, Malizos KN, Poultsides L, Tsezou A. Protective effect of atorvastatin in cultured osteoarthritic chondrocytes. J Orthop Res. 2010;28(1):110-115.

37. $\mathbf{K}$ Siddiqui $\mathbf{M}$, Maroteau C, Veluchamy $\mathbf{A}$, et al. A common missense variant of LILRB5 is associated with statin intolerance and myalgia. Eur Heart $\mathrm{J}$. 2017;38(48):3569-3575.

38. Collins R, Reith C, Emberson J, et al. Interpretation of the evidence for the efficacy and safety of statin therapy. Lancet. 2016;388(10059):2532-2561.

39. Terabe K, Takahashi N, Cobb M, Askew E, Knudson C, Knudson W. Simvastatin promotes restoration of chondrocyte morphology and phenotype. Arch Biochem Biophys. 66:1-11.

40. Yu S, Han Y, Kim S. Simvastatin abolishes nitric oxide- and reactive oxygen speciesinduced cyclooxygenase-2 expression by blocking the nuclear factor $\kappa \mathrm{B}$ pathway in rabbit articular chondrocytes. Cell Biol Int. 10:2153-2162.

41. Dijkmans PA, Juffermans LJM, Musters RJP, et al. Microbubbles and ultrasound: From diagnosis to therapy. Eur J Echocardiogr. 2004;5(4):245-256.

42. Al-Jawadi S, Thakur SS. Ultrasound-responsive lipid microbubbles for drug delivery: A review of preparation techniques to optimise formulation size, stability and drug loading. Int $J$ Pharm. 2020;585:119559.

43. Liang H. D, Tang J, Halliwell M. Sonoporation, drug delivery, and gene therapy. Proc Inst Mech Eng H. 2010;224(2):343-361.

44. Liu F, Zhu J, Huang Y, et al. Hypolipidemic effect of SR-BI gene delivery by combining cationic liposomal microbubbles and ultrasound in hypercholesterolemic rats. Mol Med Rep. 2013;7(6):1965-1969.

45. Lu QL, Liang H. D, Partridge T, Blomley MJK. Microbubble ultrasound improves the efficiency of gene transduction in skeletal muscle in vivo with reduced tissue damage. Gene Ther. 2003;10(5):396-405

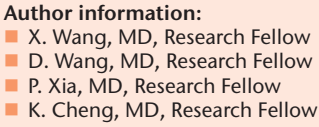

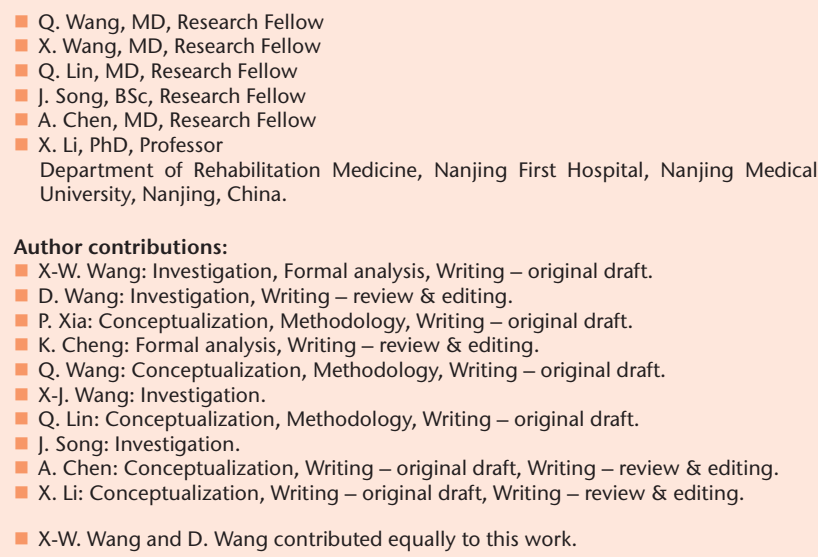
Number 81772437,82072541 ). No benefits in any form have been received or will be received from a commercial party related directly or indirectly to the subject of this article.

Acknowledgements:

We thank the National Natural Science Foundation of China (No. 81772437, 82072541) for financial support.

Ethical review statement:

The experimental protocol relating to rabbits was approved by the Nanjing Medical University Ethics Committee of Nanjing Hospital (20150829).

Open access funding

The authors confirm that the open access funding for this study was provided by the National Natural Science Foundation of China.

(c) 2021 Author(s) et al. This is an open-access article distributed under the terms of the Creative Commons Attribution Non-Commercial No Derivatives (CC BY-NC-ND 4.0) (t) the original author and source are credited. See https://creativecommons.org/licenses/ by-nc-nd/4.0/. 